\title{
ResearchOnline@JCU
}

This is the Accepted Version of a paper published in the Journal: European Journal of Applied Physiology

Stanley, Jamie, Halliday, Aaron, D'Auria, Shaun, Buchheit, Martin, and Leicht, Anthony S. (2015) Effect of sauna-based heat acclimation on plasma volume and heart rate variability. European Journal of Applied Physiology, 115 (4). pp. 785-794.

http://dx.doi.org/10.1007/s00421-014-3060-1

(C) 2015. This manuscript version is made available under the CC-BY-NC-ND 4.0 license http://creativecommons.org/licenses/by-nc-nd/4.0/

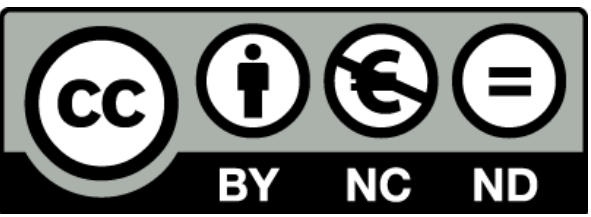




\section{Effect of sauna-based heat acclimation on plasma volume and heart rate variability}

Jamie Stanley ${ }^{1,2}$, Aaron Halliday ${ }^{3}$, Shaun D’Auria ${ }^{4}$, Martin Buchheit ${ }^{5}$, Anthony S. Leicht ${ }^{3}$

${ }^{1}$ Centre of Excellence for Applied Sport Science Research, Queensland Academy of Sport, Brisbane, Australia

${ }^{2}$ School of Human Movement Studies, University of Queensland, Brisbane, Australia

${ }^{3}$ College of Healthcare Sciences, James Cook University, Townsville, Australia

${ }^{4}$ Triathlon Program, Queensland Academy of Sport, Brisbane, Australia

${ }^{5}$ Sport Science Unit, Myorobie Association, Montvalezan, France

\section{Corresponding author:}

Jamie Stanley, School of Human Movement Studies, The University of Queensland, Brisbane, Queensland 4072, Australia; E-mail: j.stanley@uq.edu.au.

Total word count: 5285 words (excluding abstract, references, tables, figures, acknowledgements, etc)

Abstract word count: 248 words

Number of references: $\quad 39$

Number of tables: $\quad 1$

Number of figures: $\quad 4$ 


\begin{abstract}
Purpose: We investigated the effect of post-exercise sauna bathing on plasma volume (PV) expansion and
\end{abstract} whether such responses can be tracked by changes in heart rate (HR) based measures.

Methods: Seven, well-trained, male cyclists were monitored for 35 consecutive days (17 d baseline training, $10 \mathrm{~d}$ training plus sauna, $8 \mathrm{~d}$ training). Sauna exposure consisted of $30 \mathrm{~min}\left(87^{\circ} \mathrm{C}, 11 \%\right.$ relative humidity) immediately following normal training. Capillary blood samples were collected to assess PV changes while resting seated. $\mathrm{HR}\left(\mathrm{HR}_{\text {wake }}\right)$ and vagal-related $\mathrm{HR}$ variability (natural logarithm of square-root mean squared differences of successive $\mathrm{R}-\mathrm{R}$ intervals, $\ln \mathrm{rMSSD_{ \text {wake } }}$ ) were assessed daily upon waking. A sub-maximal cycle test $(5 \mathrm{~min}$ at $125 \mathrm{~W})$ was performed on days $1,8,15,22,25,29$, and 35 and $\mathrm{HR}$ recovery $\left(\mathrm{HRR}_{60 \mathrm{~s}}\right)$ and $\mathrm{ln}$ $\mathrm{rMSSD}_{\text {postex }}$ were assessed post-exercise. Effects were examined using magnitude-based inferences.

Results: Compared with baseline, sauna resulted in: 1) peak PV expansion after 4 exposures with a likely large increase $\left[+17.8 \%\left(90 \%\right.\right.$ confidence limits, 7.4;29.2)]; 2) reduction of $\mathrm{HR}_{\text {wake }}$ by a trivial-to-moderate amount $[-10.2 \%(-15.9 ;-4.0)] ; 3)$ trivial-to-small changes for $\ln \mathrm{rMSSD}_{\text {wake }}[4.3 \%(1.9 ; 6.8)]$ and $\ln \mathrm{rMSSD}_{\text {postex }}[-2.4 \%$ $(-9.1 ; 4.9)]$; and 4) a likely moderate decrease in $\operatorname{HRR}_{60 \mathrm{~s}}[-15.6 \%(-30.9 ; 3.0)]$. Correlations between individual changes in PV and HR measures were all unclear.

Conclusions: Sauna-bathing following normal training largely expanded PV in well-trained cyclists after just 4 exposures. The utility of HR and HRV indices for tracking changes in PV was however uncertain. Future studies will clarify mechanisms and performance benefits of post-training sauna bathing.

Keywords: heat exposure; blood volume; cardiac parasympathetic activity; post-exercise; cyclists 


\section{Abbreviations}

\begin{tabular}{|c|c|}
\hline $\mathrm{CV}$ & Coefficient of variation \\
\hline $\mathrm{ES}$ & Effect size \\
\hline $\mathrm{Hb}$ & Haemoglobin \\
\hline Hct & Haematocrit \\
\hline HR & Heart rate \\
\hline $\mathrm{HR}_{\mathrm{ex}}$ & Heart rate during the 5-min submaximal $(125 \mathrm{~W})$ exercise test \\
\hline HRR & Heart rate recovery \\
\hline $\mathrm{HRR}_{60 \mathrm{~s}}$ & Heart rate recovery at 60 seconds post-exercise \\
\hline HRV & Heart rate variability \\
\hline $\mathrm{HR}_{\text {wake }}$ & Heart rate upon waking \\
\hline $\ln \mathrm{rMSSD}_{\text {postex }}$ & Natural logarithm of the rMSSD following submaximal exercise \\
\hline In $\mathrm{rMSSD}_{\text {wake }}$ & Natural logarithm of the rMSSD upon waking \\
\hline PV & Plasma volume \\
\hline rMSSD & $\begin{array}{l}\text { Square root mean of the sum of the squared differences between adjacent normal } \\
R-R \text { intervals }\end{array}$ \\
\hline SWC & Smallest worthwhile change \\
\hline YoYoIR2 & Yo-Yo Intermittent recovery level 2 test \\
\hline
\end{tabular}




\section{Introduction}

In an increasingly competitive elite sporting environment, identifying methods to extract additional marginal performance gains from already demanding training schedules is of importance to athletes and coaches. Recently, supplementing training with heat acclimation has garnered increasing interest (Garrett et al. 2012; Garrett et al. 2009; Garrett et al. 2011; Lorenzo et al. 2010) primarily because the physiological adaptations including expansion of blood plasma volume (PV) may contribute to improved myocardial efficiency (Horowitz et al. 1986b), increased ventricular compliance (Horowitz et al. 1986a), and improved maximal cardiac output (Lorenzo et al. 2010) — all of which translate into improved physical performance in all (i.e., cool, temperate, and hot) environmental conditions (Racinais et al. 2014).

Traditionally, heat acclimation has involved exercising in $35-45^{\circ} \mathrm{C}, 10-90 \%$ relative humidity for up to 90 min for 8-22 consecutive days (Garrett et al. 2011). In highly-trained athletes, heat acclimation elicited increased PV $(6.5 \%$; effect size $[\mathrm{ES}]=2.0)($ Lorenzo et al. 2010), increased maximal aerobic power in cool $(4.9 \% ; \mathrm{ES}=1.5)$ and hot $(8.1 \% ; \mathrm{ES}=2.0)$ conditions (Lorenzo et al. 2010), increased lactate threshold in cool $(5.1 \%, \mathrm{ES}=0.3)$ and hot $(2.9 \% ; \mathrm{ES}=0.1)$ conditions (Lorenzo et al. 2010), improved time-trial performance in cool $(6.2 \% ; \mathrm{ES}=$ $1.1)$ and hot $(8.0 \% ; \mathrm{ES}=1.2)$ conditions (Lorenzo et al. 2010), and increased the distance covered $(42 \%, \mathrm{ES}=$ 2.0) during intermittent exercise performance in hot conditions (Racinais et al. 2014). Similar physiological and performance adaptations have been demonstrated following shorter durations of heat acclimation. For example, 5 days of exercising in the heat (total heat exposure of $3 \mathrm{~h} 45 \mathrm{~min}$ ) induced a $4.5 \%(\mathrm{ES}=0.8)$ increase in PV accompanied with a $1 \%(\mathrm{ES}=0.3)$ improvement time-trial performance in highly-trained rowers (Garrett et al. 2012). Similarly, non-heat-acclimatised semi-professional soccer players demonstrated a 5.7\% $(\mathrm{ES}=1.2)$ increase in PV accompanied with a 7\% $(\mathrm{ES}=0.5)$ increase in intermittent exercise performance in cool conditions following 6 days (total heat exposure of $7 \mathrm{~h} 30 \mathrm{~min}$ ) of training in the heat (Buchheit et al. 2011).

Heat acclimation is not without limitations. For athletes living in temperate climates, heat acclimatization/acclimation necessitates traveling to a location with the appropriate conditions for a specific training block (Buchheit et al. 2011; Racinais et al. 2014), or alternatively training in a climate chamber (Garrett et al. 2012; Lorenzo et al. 2010). Both options are expensive, unfeasible in certain situations (e.g., training with the ball in a climate chamber would be impossible for team sport athletes), and may compromise the quality of training (Garrett et al. 2011). One study has demonstrated that the acute physiological responses to heat were 
augmented when sauna bathing followed exercise (Ridge and Pyke 1986). Therefore, exposure to higher temperatures such as those experienced during sauna bathing $\left(>50^{\circ} \mathrm{C}\right)$ immediately following training may offer a more efficient means to stimulate ergogenic benefits with minimal disturbance to training. Scoon et al. (2007) had trained runners bathe in a sauna for $~ 30$-min immediately following training on $12-15$ occasions over 3 weeks and demonstrated a $7.1 \%(\mathrm{ES}=1.3)$ difference in PV and $1.9 \%$ estimated improvement in $5 \mathrm{~km}$ time-trial performance compared with a 3-week period of normal training. The difference in PV and performance were near perfectly correlated $(\mathrm{r}=-0.96)$ (Scoon et al. 2007).

To date however, the time course of sauna-induced changes in PV has not been examined. Heart rate based measures may provide a simple, non-invasive tool to monitor changes in PV that are expected with sauna bathing (Buchheit 2014; Stanley et al. 2013b). For example, following traditional heat acclimation (accompanied with PV expansion), HR during (Buchheit et al. 2011; Garrett et al. 2012; Lorenzo et al. 2010) sub-maximal exercise is reduced, HR variability (HRV) at rest (Buchheit et al. 2011) and during immediate post-exercise recovery (Buchheit et al. 2009) is increased, while HR recovery (HRR) is unaffected (Buchheit et al. 2009; Buchheit et al. 2011). Changes in the aforementioned HR measures also demonstrate moderate-to-very large correlations with increased PV (Buchheit et al. 2009; Buchheit et al. 2011) and improvements in exercise performance (Buchheit 2014; Buchheit et al. 2013; Buchheit et al. 2011; Racinais et al. 2014). Most importantly however, because the magnitude of HR responses after just one day in the heat are similar to those expected after a month of normal training (Buchheit et al. 2013), changes in HR-based measures during heat acclimation appear more likely a consequence of expanded PV rather than actual changes in fitness/fatigue. To date, it is not known how HR-based indexes respond to sauna-based heat acclimation, or which indexes are most sensitive to the induced physiological adaptations. Therefore, the aim of this study was to 1) investigate the time course of PV changes during and after a post-training sauna intervention, and 2) determine how and/or which HR-based measures can be used to monitor these changes.

\section{Methods}

\section{Participants}

Seven endurance-trained male cyclists participated in this study (age $23.3 \pm 4.0$ years, height $1.79 \pm 0.07 \mathrm{~m}$, body mass $78.0 \pm 6.6 \mathrm{~kg}$, body fat $10.2 \pm 1.9 \%, \mathrm{VO}_{2 \max } 60.4 \pm 4.7 \mathrm{~mL} \cdot \mathrm{kg}^{-1} \mathrm{~min}^{-1}$, peak HR $193 \pm 10$ beats.min $^{-1}$, peak power output $427 \pm 38 \mathrm{~W}$ ). All participants had trained consistently for at least 6 months prior to the study, 
were not taking iron supplementation, had not donated blood within 3 months prior to testing and had no prior history of heat related illnesses. All participants completed questionnaires to confirm their health status and provided written informed consent in accordance with approval by the Human Research Ethics Committee of James Cook University.

\section{Experimental Design}

An overview of the interrupted time series study design is displayed in Figure 1. The study consisted of presauna (days 1-17), sauna (days 18-28) and post-sauna (days 29-35) stages. The cyclists' $\mathrm{VO}_{2 \max }$, peak HR and peak power output was determined in the week prior to the pre-sauna period, and during the week following the completion of the monitoring period (i.e., post day 35) using a graded cycling protocol commencing at an initial workload of $175 \mathrm{~W}$ that increased by $25 \mathrm{~W}$ per minute until volitional exhaustion. Expired air was collected via a one-way mouthpiece (Hans Rudolph Inc, Shawnee, Kansas) and analysed breath-by-breath with 10-s averages examined for $\mathrm{VO}_{2 \max }$ attainment. Peak HR was the highest HR recorded during the final $10 \mathrm{~s}$ of the test. Peak power output was calculated as the power output from the last completed stage of the incremental exercise test, plus the fraction of time spent in the next stage multiplied by $25 \mathrm{~W}$. The cyclists bathed in a sauna $\left(80-90^{\circ} \mathrm{C}\right)$ following training on ten consecutive days. For the duration of the study, the cyclists completed their own training and recorded their training duration and subjective intensity (session RPE) for each training session (Wallace et al. 2013). The cyclists were instructed to replicate the training performed during days 1-17 during days 18-35. Each day upon waking, the cyclists adopted a seated position to record HR. The cyclists completed a submaximal cycling test periodically for the duration of the study and HR was recorded during the acute recovery period (Figure 1). Capillary blood samples were collected and evaluated for PV changes and sauna responses (Figure 1).

[Insert Figure 1 here]

\section{Sauna Bathing}

Cyclists attended a sauna facility between 5:30 p.m. and 7:00 p.m. immediately after or during the later part (i.e. warm-down) of daily training sessions for ten consecutive days (Figure 1). The cyclists remained in the sauna for $30 \mathrm{~min}$ in a seated upright position. We opted to standardize the duration of sauna exposure rather than standardize the relative heat stress to minimize the potential impact on the cyclists' daily life/training schedule 
and best replicate what can be feasibly implemented in the field. Before, after, and every 5 min during sauna bathing, tympanic temperature (Welch-Allyn 6021, Braun, Germany), blood pressure (ALP K2 Aneroid, Japan), thermal comfort $(1=$ comfortable to $5=$ extremely uncomfortable $)$ and sensation $(1=$ unbearably cold to $13=$ unbearably hot) were measured. Environmental conditions within the sauna were measured using a portable weather station (Kestral 4000, Boothwyn, Pennsylvania). Dry nude body mass was measured prior to and following each sauna session with cyclists instructed to drink $150 \%$ of fluid difference within $6 \mathrm{~h}$ following the sauna (Shirreffs et al. 2004).

$\downarrow$ Submaximal exercise test

- Capillary blood and urine sample

* Seated HRV recording

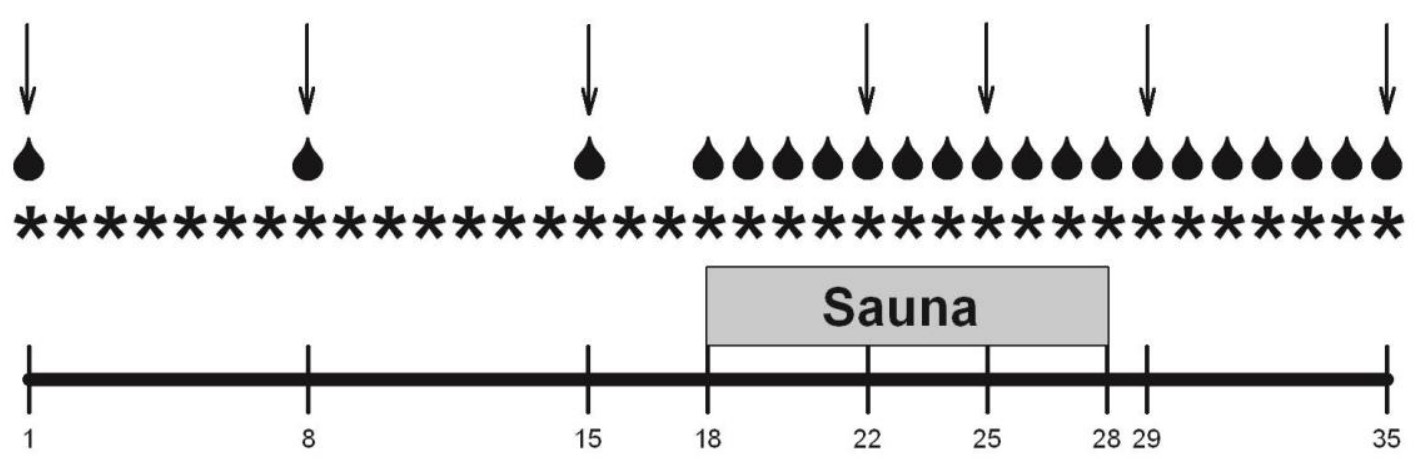

Figure 1 Overview of the study design

\section{Days}




\section{Hydration status and plasma volume assessment}

On the morning of the experimental trials, the cyclists collected a urine sample (mid-stream), and entered the laboratory (in a fasted state) between 5:00 a.m. and 6:30 a.m. The specific gravity of each sample was measured using a digital refractometer (UG-a Atago, Honcho, Japan). The cyclists were considered hydrated if the urine specific gravity was $\leq 1.030$ g.mL $\mathrm{mL}^{-1}$ (Armstrong et al. 1994). Capillary blood samples were collected from multiple fingers from day-to-day to ensure adequate blood was extracted. The cyclists remained in a seated position for at least 10 min prior to blood sampling. Each site was cleansed with an alcohol wipe, dried and a sterile lancet applied. The first drop of blood was discarded with blood collected into duplicate $100 \mu \mathrm{L}$ heparinised capillary tubes for haematocrit (Hct) determination and microcuvettes for haemoglobin $(\mathrm{Hb})$ determination. Samples were collected on days 1, 8, and 15, and days 18-35. Blood samples were analysed for $\mathrm{Hb}$ via a DHT Portable Haemoglobinometer with a reported coefficient of variation of $2.3 \pm 0.7 \%$ (SanchisGomar et al. 2013). If there was a difference of $>5 \%$ in measured $\mathrm{Hb}$ between samples, a third blood sample was collected and analysed. Blood samples for Hct were centrifuged for 5 min at $14000 \mathrm{rpm}$, and assessed for Hct by 2 independent reviewers (Morris et al. 1999). Changes in PV (\%) were calculated from the following equation (Strauss et al. 1951):

$$
=100 \times \frac{\mathrm{Hb}(\text { before })}{\mathrm{Hb}(\text { after })} \times \frac{1-\mathrm{Hct}(\text { after })}{1-\mathrm{Hct}(\text { before })}-100
$$

Where before is the concentration at pre-sauna and after is the concentration during or following the sauna intervention. All blood analysis was performed immediately onsite.

\section{Sub-maximal Performance Test}

On days $1,8,15,22,25,29$ and 35 , following the blood sampling procedures, the cyclists performed a submaximal cycle test (Monark 828E, Varberg, Sweden) at a workload of $125 \mathrm{~W}$ and cadence of $100 \mathrm{rpm}$ for $5 \mathrm{~min}$ (Buchheit et al. 2010). Heart rate was recorded during the $5 \mathrm{~min}$ of exercise $\left(\mathrm{HR}_{\mathrm{ex}}\right)$. Immediately upon completion, the cyclists dismounted and remained seated for $10 \mathrm{~min}$ on a chair located adjacent to the ergometer for recovery measurements.

\section{Heart Rate Variability}


Upon waking each morning during the study, HR was recorded (Ambit 2, Suunto Oy, Vantaa, Finland) for 7 min in a seated position. $R-R$ interval data files were transferred to a computer and further signal processing was performed using a dedicated HRV analysis program (Kubios HRV Analysis version 2.0 beta 1, The Biomedical Signals Analysis Group, University of Kuopio, Finland) (Stanley et al. 2013a). Each file was visually inspected. Artifacts were manually removed and occasional ectopic beats were automatically replaced with the interpolated adjacent $R-R$ interval values.

Heart rate and the natural logarithm of the square root mean of the sum of the squared differences between adjacent normal $R-R$ intervals (ln rMSSD) was calculated from the last $5 \mathrm{~min}$ of the 7 -min (seated) waking recordings $\left(\mathrm{HR}_{\text {wake }}\right.$, $\left.\ln \mathrm{rMSSD}_{\text {wake }}\right)$ and from the last $3 \mathrm{~min}$ of the $5 \mathrm{~min}$ following the sub-maximal performance test $\left(\mathrm{HR}_{\text {postex }}\right.$, ln $\left.\mathrm{rMSSD}_{\text {postex }}\right)$ (Stanley et al. 2013a) to provide an index of cardiac parasympathetic activity (Task Force of the European Society of Cardiology and the North American Society of Pacing and Electrophysiology 1996). Analysis was restricted to ln rMSSD due to greater reliability compared with spectral indices (Al Haddad et al. 2011) during ambulatory trials of variable respiration rate (Penttilä et al. 2001).

\section{Data and Statistical Analysis}

All data in the text and figures are presented as mean with the $90 \%$ confidence interval (CI) unless otherwise stated. The practical significance of data was assessed via magnitude-based inferences (Hopkins et al. 2009). A qualitative approach was selected as traditional statistical approaches generally fail to indicate the magnitude of an effect, which is more relevant to athletic performance (Hopkins 2010). All data were log-transformed before analysis to reduce bias arising from non-uniformity in error (Hopkins et al. 2009).

Because of the interrupted time series study design, comparisons were made between the mean response of each variable during the pre-sauna period (days 1-18) and the response during each day after sauna treatment commenced (days 19-35) using a modified statistical spreadsheet (Hopkins 2006). Comparisons for physiological responses were not made for a particular day if data for $>3$ athletes were lost/excluded due to equipment malfunction and/or the presence of artefacts. The spreadsheets calculate the within-trial standardized differences or effect sizes (ES, 90\% CI) using the pooled standard deviation (Cohen 1988). The following threshold values for ES were employed: $<0.2$ as trivial, $>0.2$ as small, $>0.6$ as moderate, $>1.2$ as large, $>2.0$ as very large, and $>4.0$ as extremely large (Hopkins et al. 2009). Additionally, we calculated the probabilities of 
whether the true (unknown) differences were lower, similar or higher than the smallest worthwhile change or difference. The smallest worthwhile change/difference for all variables was calculated from the day-to-day variation during the pre-sauna period (e.g. days 1-18) expressed as the coefficient of variation (CV) (Hopkins 2000). The natural daily variation during the pre-sauna period expressed as the CV (90\% CI) was 64.8\% (50.9; 92.4) for training duration, $37.6 \%(30.2 ; 51.5)$ for training intensity, $102.0 \%(78.8 ; 149.9)$ for training load, $5.5 \%(4.1 ; 9.4)$ for $\mathrm{PV}, 8.6 \%(7.5 ; 10.2)$ for $\mathrm{HR}_{\text {wake }}, 6.6 \%(5.8 ; 7.8)$ for $\ln \mathrm{rMSSD}_{\text {wake }}, 14.1 \%(10.3 ; 24.7)$ for

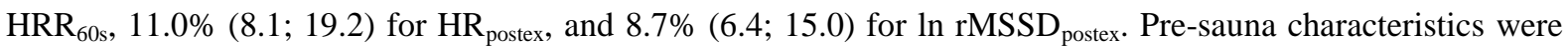
included as a covariate for within-trial analysis of training duration, rating and load. Age was included as a covariate for HRV responses due to its reported effect on baseline and training-induced HRV responses (Sandercock et al. 2005). The qualitative chances of either higher or lower difference were evaluated as follows: $1 \%$, almost certainly not; $1-5 \%$, very unlikely; 5-25\%, unlikely; 25-75\%, possible; 75-95\%, likely; 95-99\%, very likely; >99\%, almost certain. If the chances of a substantially higher or lower difference were both $>5 \%$, the true difference was assessed as 'unclear' (Hopkins et al. 2009). Pearson's product moment correlation analysis examined the association between individual percentage changes in PV and HR based variables. The following criteria were adopted to interpret the magnitude of the correlation $(r)$ between these measures: $<0.1$ as trivial; $0.1-0.3$ as small; $>0.3-0.5$ as moderate; $>0.5-0.7$ as large; $>0.7-0.9$ as very large; and $>0.9-1.0$ as almost perfect (Hopkins et al., 2009). If the $90 \%$ CI overlapped small positive and negative values, the magnitude of the correlation was deemed 'unclear'; otherwise, the magnitude of the correlation was deemed to be the observed magnitude (Hopkins et al., 2009).

\section{Results}

Approximately $0-13 \%$ of data (i.e. HR, HRV, PV, etc.) were lost/excluded from analysis due to equipment malfunction and/or the presence of artefacts. These events were random in occurrence and unlikely biased any statistical analysis. However, comparisons for $\mathrm{HR}_{\text {wake }}$ and $\ln \mathrm{rMSSD}_{\text {wake }}$ on days 19 and 26 were excluded from analysis because data for $>3$ athletes were lost/excluded due to equipment malfunction and/or the presence of artefacts. All athletes presented to the laboratory in a hydrated state as indicated by a mean urine specific gravity (SD) of $1.019(0.007) \mathrm{g} \cdot \mathrm{mL}^{-1}$.

During the pre-sauna period, mean \pm SD values were $74 \pm 11$ min training duration, $6 \pm 1$ for training intensity, $446 \pm 82$ arbitrary units for training load, $94.9 \pm 8.1 \%$ of day 1 value for PV, $59 \pm 6$ beats.min ${ }^{-1}$ for $\mathrm{HR}_{\mathrm{wake}}, 4.1$ 
$\pm 0.4 \mathrm{~ms}$ for $\ln \mathrm{rMSSD}_{\text {wake }}, 79 \pm 15$ beats for $\mathrm{HRR}_{60 \mathrm{~s}}, 69 \pm 10$ beats.min ${ }^{-1}$ for $\mathrm{HR}_{\text {postex }}$, and $3.6 \pm 0.3 \mathrm{~ms}$ for $\mathrm{ln}$ $\operatorname{rMSSD}_{\text {postex }}$

Each sauna exposure was perceived as hot and very uncomfortable, and was accompanied by an increase in tympanic temperature and loss of weight due to sweat loss (Table 1).

Individual and mean training load distribution for the entire study is presented in Figure 2a. Prior to and following the sauna period, daily training loads were similar, however, training duration and intensity was substantially reduced during the sauna period (Figure $2 b, c, d$ ).

Post-exercise sauna bathing very likely increased PV (+17.8\% 90\% CL $(7.4 ; 29.2)$, ES=+1.7 90\% CL (0.7; 2.6), $\%$ chances for greater/similar/lower values of 97/3/0\%) after just 4 days of sauna bathing (Figure 3a). An initial moderate increase in $\mathrm{HR}_{\text {wake }}(+8.3 \%(-0.8 ; 18.2), \mathrm{ES}=+0.6(-0.1 ; 1.3), 48 / 52 / 0 \%)$ was observed after day 2 of sauna treatment, thereafter trivial-to-moderate reductions in $\mathrm{HR}_{\text {wake }}$ were observed peaking after 6 days of sauna bathing $(-10.2 \%(-4.0 ;-15.9)$, ES=-0.8 (-1.3;-0.3), 0/24/76\%, Fig 3b). Trivial-to-small increases in $\ln$ rMSSD $_{\text {wake }}$ were observed after day 3 of sauna treatment (Fig 3c).

Compared with pre-sauna values, changes in $\mathrm{HR}_{\mathrm{ex}}$ remained trivial or unclear until the $7^{\text {th }}$ day following sauna treatment after which a likely moderate increase was observed $(+5.5 \%(2.8 ; 8.3), \mathrm{ES}=0.7(0.3 ; 1.0), 90 / 10 / 0 \%$, Fig 4a). $\mathrm{HRR}_{60 \mathrm{~s}}$ and $\mathrm{ln} \mathrm{rMSSD}_{\text {postex }}$ demonstrated likely moderate reductions and trivial-to-small reductions, respectively, after commencement of sauna treatment compared with pre-sauna values (Fig 4b, c). There were no clear changes in peak HR $(-0.1 \%(-1.7 ; 1.4), \mathrm{ES}=-0.2(-0.27 ; 0.23), 7 / 82 / 11 \%)$ or peak power output $(0.4 \%$ $(-2.1 ; 3.0), \mathrm{ES}=0.04(-0.21 ; 0.29), 34 / 50 / 16 \%)$ from the beginning to the end of the study.

Correlations between individual changes in PV and individual changes in $\operatorname{HR}_{\text {wake }}[\mathrm{r}=-0.01(-0.17 ; 0.15), \mathrm{n}=$ 103], In $\mathrm{rMSSD}_{\text {wake }}[\mathrm{r}=0.04(-0.13 ; 0.20), \mathrm{n}=103], \mathrm{HRR}_{60 \mathrm{~s}}[\mathrm{r}=0.09(-0.23 ; 0.40), \mathrm{n}=28], \mathrm{HR}_{\text {postex }}[\mathrm{r}=0.05$ $(-0.30 ; 0.38), \mathrm{n}=25]$, or $\ln \mathrm{rMSSD}_{\text {postex }}[\mathrm{r}=-0.10(-0.42 ; 0.25), \mathrm{n}=25]$ were all unclear. Large correlations were observed between individual changes in $\mathrm{HR}_{\text {wake }}$ and individual changes in $\ln \mathrm{rMSSD}_{\text {wake }}[\mathrm{r}=-0.66(90 \%$ $\mathrm{CI}-0.75 ;-0.56), \mathrm{n}=103]$ and individual changes in $\mathrm{HR}_{\text {postex }}$ and individual changes in $\ln \mathrm{rMSSD}_{\text {postex }}[\mathrm{r}=$ $-0.68(-0.83 ;-0.45), \mathrm{n}=25]$. There were no clear correlations observed between individual changes in $\mathrm{HRR}_{60 \mathrm{~s}}$ 
and individual changes in $\mathrm{HR}_{\text {postex }}[\mathrm{r}=0.32(-0.02 ; 0.59), \mathrm{n}=25]$ or individual changes in ln $\mathrm{rMSSD}_{\text {postex }}[\mathrm{r}=$ $0.02(-0.32 ; 0.36), \mathrm{n}=25]$.

Table 1 Participant and environmental characteristics prior to (Pre-Sauna), during (Sauna) and following (Postsauna) the sauna bathing period.

\begin{tabular}{|c|c|c|c|}
\hline & Pre-Sauna & Sauna & Post-Sauna \\
\hline Sauna temperature $\left({ }^{\circ} \mathrm{C}\right)$ & & $87.0 \pm 13.7$ & \\
\hline Sauna humidity (\%) & & $10.7 \pm 12.6$ & \\
\hline Tympanic temperature $\left({ }^{\circ} \mathrm{C}\right)$ & & $39.2 \pm 1.1$ & \\
\hline Thermal sensation & & $10.6 \pm 1.0$ & \\
\hline Thermal comfort & & $3.9 \pm 0.5$ & \\
\hline Sweat rate/loss $\left(\mathrm{kg} \cdot \mathrm{hr}^{-1}\right)$ & & $1.6 \pm 0.8$ & \\
\hline \multicolumn{4}{|l|}{ Outside environment: } \\
\hline Maximum temperature $\left({ }^{\circ} \mathrm{C}\right)$ & $27.7 \pm 1.4$ & $27.7 \pm 0.6$ & $29.4 \pm 1.3$ \\
\hline Minimum temperature $\left({ }^{\circ} \mathrm{C}\right)$ & $15.4 \pm 2.2$ & $16.8 \pm 1.8$ & $18.1 \pm 2.0$ \\
\hline Humidity (\%) & $58.0 \pm 10.9$ & $51.7 \pm 7.0$ & $56.3 \pm 10.6$ \\
\hline
\end{tabular}

Values are mean \pm SD. 

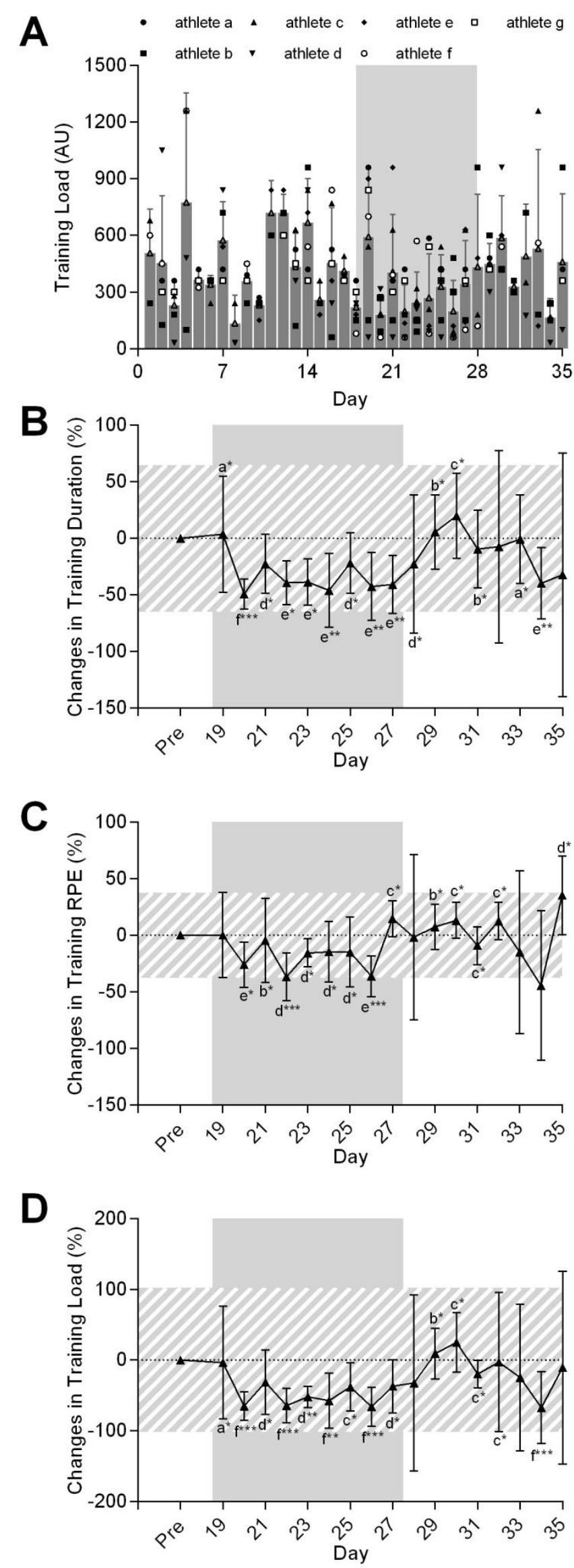

Figure 2 Distribution of individual (symbols) and mean \pm SD (dark grey bars) daily training load for the duration of the study (A), change $(\% \pm 90 \%$ confidence limit) in training duration (B), training intensity (C), and training load (D) compared with mean pre-sauna exposure values. The sauna exposure period is denoted by the dark grey area. Within-trial changes are expressed qualitatively (Hopkins et al. 2009). The grey hatched area represents the smallest worthwhile change (i.e., the $\mathrm{CV}$ for each variable, see "Methods"). Standardized differences: $a=$ trivial $\mathrm{b}=$ small $; \mathrm{c}=$ moderate $; \mathrm{d}=$ large $\mathrm{e}$ $=$ very large; $\mathrm{f}=$ extremely large. Likelihood of effect: $*=$ trivial; $* *=$ possibly higher or lower; $* * *=$ likely higher or lower. 


\section{Discussion}

In the present study we investigated the time course of PV changes during and after a post-training sauna intervention, and determined how and which HR-based measures can be used to monitor these changes. The main findings of this study were as follows. First, $\sim 30$ min of sauna bathing immediately following normal daily training induced moderate-to-large increases in PV following 6 exposures (peaked after 4 exposures); PV trended back towards pre-sauna levels with subsequent sauna exposures. Second, sauna exposure elicited trivialto-moderate reductions in resting waking HR and trivial-to-small increases in resting waking vagal-related HRV that were maintained thereafter. Third, HRR following a standardised sub-maximal performance test was moderately reduced during the sauna exposure period. Last, there was no correlation between individual changes in PV and changes in any HR-based measure. Despite the lack of a 'true' control group, all comparisons were made with respect to the mean values and the natural variation measured during the 17 days

of normal training prior to sauna exposure (i.e., interrupted time series; see "Methods") using a progressive statistical approach (Hopkins et al. 2009). Further, with this statistical approach, our sample size is not limiting — and nevertheless comparable with previous studies (Garrett et al. 2012; Scoon et al. 2007). The importance of these findings is that, post-exercise sauna bathing may offer a time efficient means by which to stimulate heat acclimation without substantially impacting on daily athlete training. However, the value of HRbased measures to monitor changes in PV in this setting remains unclear. 
Figure 3 Change $(\% \pm 90 \%$ confidence

A

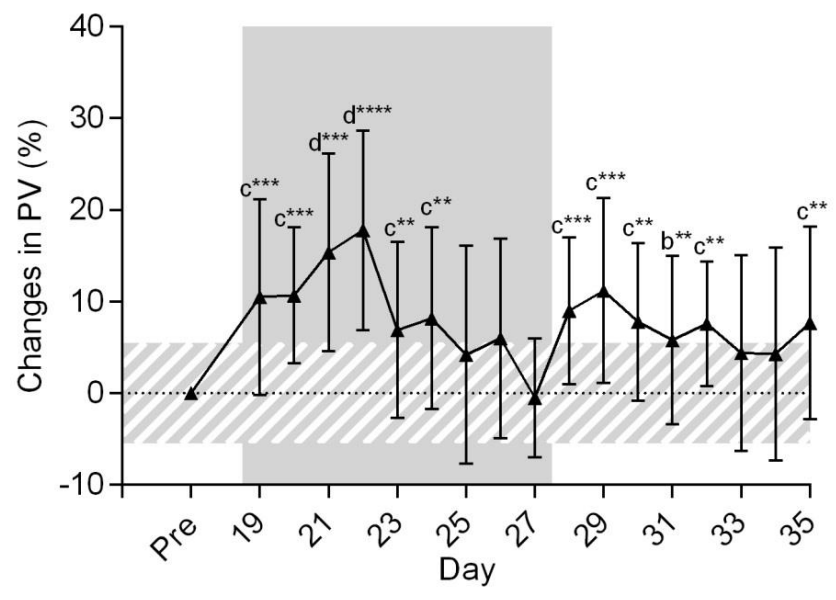

B

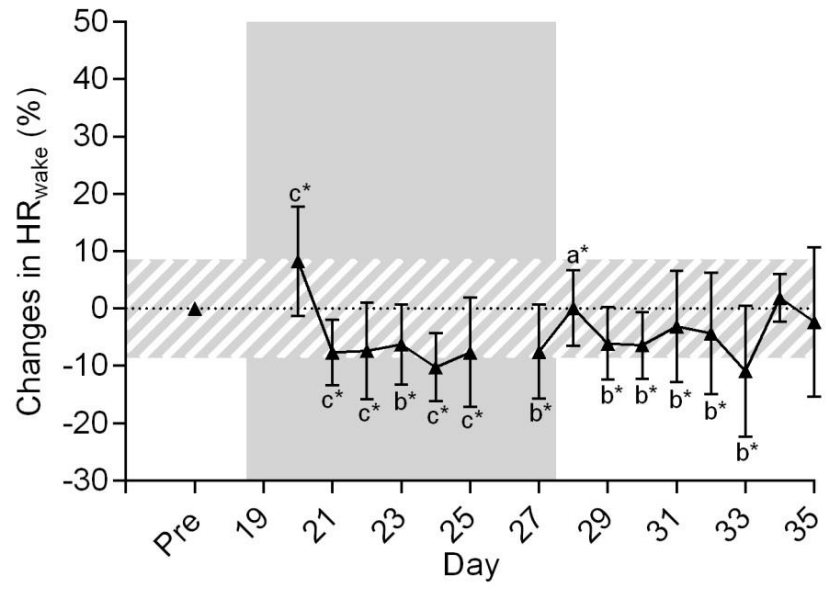

C

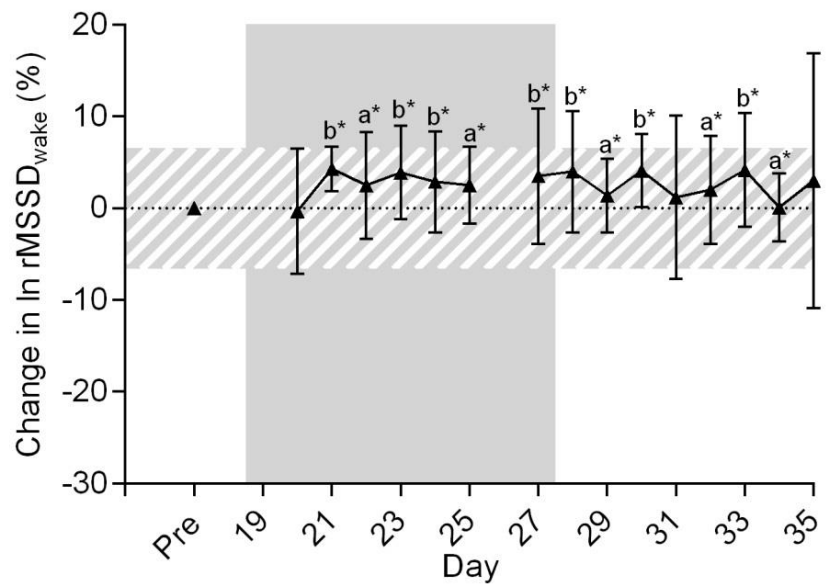

limit) in plasma volume (PV, A), seated waking heart rate $\left(\mathrm{HR}_{\text {wake }}, \mathrm{B}\right)$ and seated waking $\ln$ rMSSD (ln rMSSD ${ }_{\text {wake, }}$ C) compared with mean pre-sauna exposure values. Comparisons for $\mathrm{HR}_{\text {wake }}$ and $\mathrm{ln}$ rMSSD $_{\text {wake }}$ were excluded from analysis on days 19 and 26 because data for $>3$ athletes were lost/excluded due to equipment malfunction and/or the presence of artefacts. The sauna exposure period is denoted by the dark grey area. Within-trial changes are expressed qualitatively (Hopkins et al. 2009). The grey hatched area represents the smallest worthwhile change (i.e., the $\mathrm{CV}$ for each variable, see "Methods"). Standardized differences: $\mathrm{a}=$ trivial $\mathrm{b}=$ small; $\mathrm{c}=$ moderate; $d=$ large. Likelihood of effect: $*=$ trivial; $* *=$ possibly higher or lower; $* * *=$ likely higher or lower; $* * * *=$ very likely higher or lower. 
Figure 4 Mean change $(\% \pm 90 \%$

A

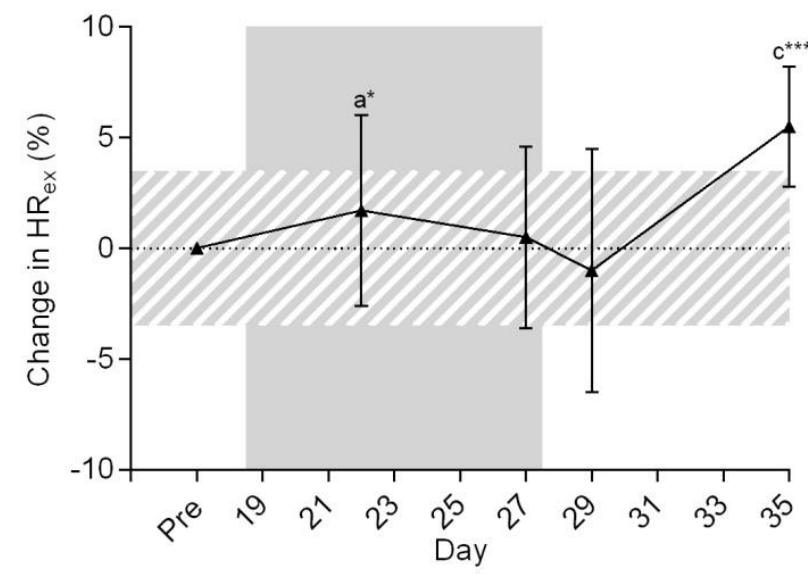

B

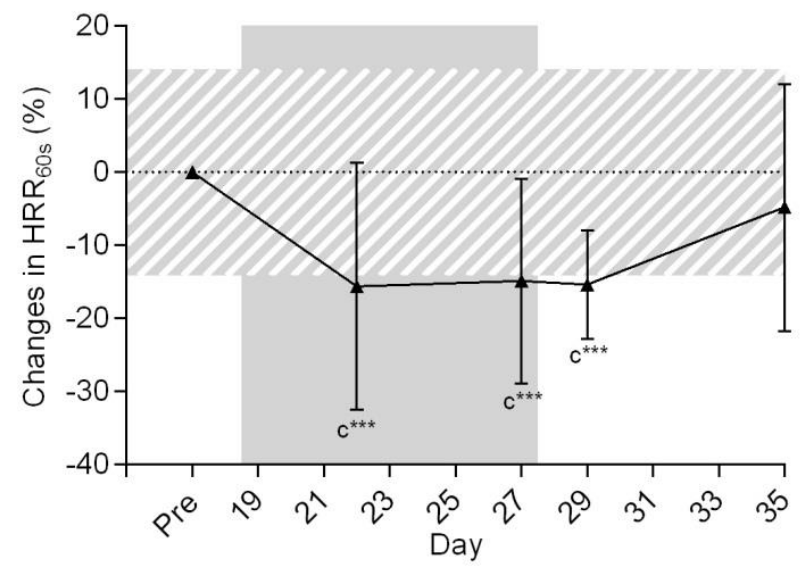

C
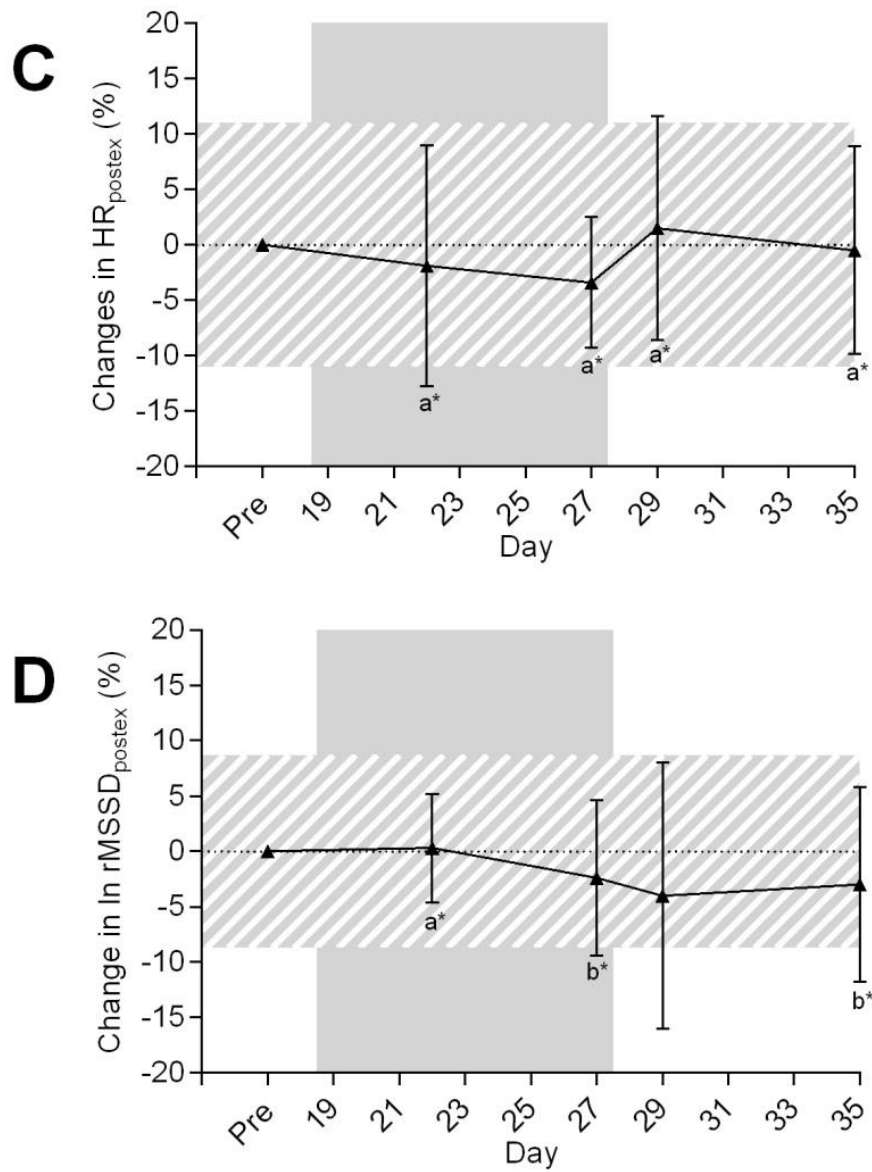

confidence limit) compared with mean pre-sauna exposure values in heart rate during a 5-min submaximal cycle test $\left(\mathrm{HR}_{\mathrm{ex}}, \mathrm{A}\right)$ heart rate recovery $60 \mathrm{~s}$ after a 5-min submaximal cycle test $\left(\mathrm{HRR}_{60 \mathrm{~s}}\right.$, B), mean heart rate during seated recovery after a 5-min submaximal cycle test $\left(\mathrm{HR}_{\text {postex }}, \mathrm{C}\right)$, and $\ln \mathrm{rMSSD}$ after a 5-min submaximal cycle test (ln rMSSD $\left._{\text {postex }}, \mathrm{D}\right)$. The sauna exposure period is denoted by the dark grey area. Within-trial changes are expressed qualitatively (Hopkins et al. 2009). The grey hatched area represents the smallest worthwhile change (i.e., the $\mathrm{CV}$ for each variable, see "Methods"). Standardized differences: $\mathrm{a}=$ trivial $\mathrm{b}=$ small; $\mathrm{c}=$ moderate. Likelihood of effect: $*=$ trivial; $* *=$ possibly higher or lower; $* * *$ $=$ likely higher or lower. 
The present study is the first to examine the time course of sauna-induced changes in PV during and following a period of post-training sauna bathing. We observed that the addition of $30 \mathrm{~min}$ of sauna exposure following normal daily training elicited physiological adaptations representative of heat acclimation. Plasma volume was largely increased $(+17.8 \%, \mathrm{ES}=1.7)$ within the first 4 days of sauna exposure $(\sim 4.5 \%$ per day $)$ compared with the mean values during 18 days of normal training pre-sauna exposure (Fig. 3a). By comparison, PV expansion following post-exercise sauna exposure $(\sim 12$ exposures over 3 weeks $)$ was large $(+7.1 \%, \mathrm{ES}=1.3)$ or $\sim 0.5 \%$ per day in well-trained runners (Scoon et al. 2007), and PV expansion following traditional heat acclimation was large $(+5.7 \%, \mathrm{ES}=1.2)$ or $\sim 1.3 \%$ per day in semi-professional soccer players following 6 days of training in the heat (Buchheit et al. 2011), moderate $(+8 \%$, ES $=0.6)$ or $\sim 1.1 \%$ per day in sub-elite cyclists following 7 days of exercising in the heat (Patterson et al. 2004), or moderate $(+4.5 \%$, ES $=0.8)$ or $\sim 0.9 \%$ per day in highlytrained rowers following 5 days of exercise in the heat (Garrett et al. 2012). Importantly, the changes in PV reported in these latter studies were not assessed daily, therefore whether greater values were reached during the interventions as in the present study remains unclear. Together, these data suggest that post-exercise sauna exposure is an equally effective and more efficient means by which to initiate PV expansion compared with traditional heat acclimation that involves training in hot conditions (Buchheit et al. 2011; Garrett et al. 2012; Garrett et al. 2009; Lorenzo et al. 2010; Patterson et al. 2004). Further, the passive nature of sauna exposure may be more beneficial for athletes because normal training, intensive training and/or specific taper sessions near to competition need not be altered (Garrett et al. 2011). Interestingly, by the $7^{\text {th }}$ sauna exposure, PV trended back towards pre-sauna levels (unclear whether values were elevated) despite subsequent sauna exposure and remained stable for the 7 days (possible-to-likely small-to-moderate elevation above pre-sauna levels) after the final sauna exposure (Fig. 3a), supporting the observation that PV expansion regresses toward baseline levels if the exercise stimulus and/or thermal load exposures remain constant (Wyndham et al. 1968). Although evidence linking PV expansion and exercise performance is not definitive (Warburton et al. 2000), the transient nature of this response highlights that periodization of sauna exposure/heat acclimation within the training/competition cycle requires consideration if intended for ergogenic benefit.

Acute increases in PV have been accompanied by reduced HR during sub-maximal exercise (Buchheit et al. 2011; Garrett et al. 2012; Lorenzo et al. 2010; Racinais et al. 2014), increased HRV at rest (Buchheit et al. 2011) and during immediate post-exercise recovery (Buchheit et al. 2009), but not HRR (Buchheit et al. 2009; Buchheit et al. 2011). Notably, changes in HR-based measures during heat acclimation appear more likely a 
consequence of expanded PV rather than changes in fitness/fatigue (Buchheit et al. 2013). Baroreflex mediated increases in cardiac parasympathetic activity (i.e., vagal-related indexes of HRV) reaches its maximal level during moderate hypervolemia but decreases with severe hypervolemia (Saitoh et al. 2008). Therefore, the trivial-to-small magnitude increases in $\ln \mathrm{rMSSD}_{\text {wake }}$ we observed after the third sauna exposure (Fig. 3c) were not surprising provided the moderate-to-large (but not very large) increase in PV (Fig. 3a). Further, the likely moderate-to-very large reduction in training load (Fig. 2) during the sauna exposure period suggests that PV expansion was probably the most likely contributor to the increase in $\ln \mathrm{rMSSD}_{\text {wake }}$ as a reduction in training load typically reduces cardiac parasympathetic activity (Buchheit 2014; Plews et al. 2013). Therefore, if training load was maintained at a level similar to that of the pre-sauna exposure period (Fig. 2), $\ln$ rMSSD $_{\text {wake }}$ would likely have been higher. Whether the self-regulated reduction in training load reflects an anticipatory mechanism or was a consequence of the added stress induced by sauna exposure, or was simply due to the extra time required for sauna bathing remains unclear. This observation nevertheless has important practical application, because any prolonged reduction in training load may impact on actual performance. Therefore to maximize ergogenic benefit, careful management of training load is required if sauna exposure/heat acclimation is to be integrated within the training/competition cycle.

Unfortunately, due to the large time commitment of the cyclists to participate in this study (maximal) cycling performance was not tested periodically throughout the monitoring period. However, the athletes did complete a second maximal test in the week following the monitoring period. No clear change in peak HR or peak power output was observed. Due to technical difficulties with gas analysis equipment we have not reported these data. Nevertheless, the cyclists performed a 5-min sub-maximal cycling test at a standardised power output periodically throughout the study (Lamberts et al. 2011). Heart rate during the exercise tests performed during the sauna exposure period was similar to values recorded during the pre-sauna exposure period-except on the final day when it was moderately elevated (Fig. 4a). The reduction in training load during the sauna exposure period possibly resulted in a reduction of the cyclists' fitness, and in turn, increased the relative exercise intensity during 5-min sub-maximal cycling test. Immediately following the 5-min sub-maximal cycling test, $\mathrm{HRR}_{60 \mathrm{~s}}$ was likely moderately reduced during and the day following the sauna exposure period (Fig. 4b). The reduction in $\mathrm{HRR}_{60 \mathrm{~s}}$ was likely not related to changes in PV (Buchheit et al. 2009; Buchheit et al. 2011), but rather due to the relative reduction in training load during the sauna exposure period (Borresen and Lambert 2007). The reasons for the lack of substantial change in $\mathrm{HR}_{\text {postex }}$ and $\ln \mathrm{rMSSD}_{\text {postex }}$ (Fig. $4 \mathrm{c}$ and $\mathrm{d}$ ) remain 
unclear, but might be linked to the fact that these indexes integrate multiple factors such as exercise intensity (Stanley et al. 2013b) and PV (Buchheit et al. 2009); therefore if one decreases while the other increases, the net response is unchanged.

A key aim of this study was to examine the utility of non-invasive HR-based measures for monitoring changes in PV. Changes in PV affect baroreflex mediated changes in cardiac parasympathetic activity (Saitoh et al. 2008) and have been largely $[\mathrm{r}=0.85(0.53 ; 0.96)]$ associated with acute changes in cardiac parasympathetic reactivation (Buchheit et al. 2009). Surprisingly, we observed no clear correlations between individual changes in PV and any HR-based index. It is possible that exercise and/or heat modified the set point of baroreceptor activation (Buchheit et al. 2009; Stanley et al. 2013b). Although not a main objective of this study, our data suggest that HR-based measures provide similar information because respective correlations between individual

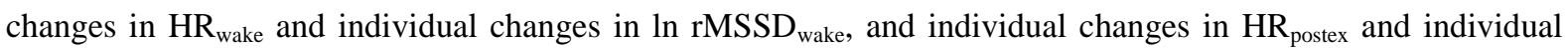
changes in $\ln \mathrm{rMSSD}_{\text {postex }}$ were large $[\mathrm{r}=-0.66(-0.75 ;-0.56)$ and $\mathrm{r}=-0.68(-0.83 ;-0.45)]$. Conversely, the lack of clear correlations between individual changes in $\mathrm{HRR}_{60 \mathrm{~s}}$ and individual changes in $\mathrm{HR}_{\text {postex }}[\mathrm{r}=0.32$ $(-0.02 ; 0.59)]$ or individual changes in $\ln \operatorname{rMSSD}_{\text {postex }}[r=0.02(-0.32 ; 0.36)]$ suggests that these indices capture different information (Buchheit et al. 2007) concerning cardiovascular control.

The current study is not without some limitations. First, changes in PV were estimated via changes in Hct and $\mathrm{Hb}$ concentrations. This method assumes the erythrocytes remain within the circulation and accurately reflects small to moderate changes $(<10 \%)$ in PV although underestimates large changes in PV by $25-30 \%$ (Lundvall and Lindgren 1998). Nevertheless, we demonstrated a coefficient of variation of $5.5 \%$ in PV measurement prior to sauna exposure and are confident that our statistical analysis provides a clear and practical indication of the sauna induced changes. Second, although the cyclists were instructed to replicate the training performed during days 1-17 during days 18-35, the training load was reduced during the sauna exposure period (Fig. 2). This self-regulated reduction in training load possibly reflects an anticipatory mechanism or was a consequence of the added stress induced by sauna exposure. Interestingly, the reduction in training volume was of comparable duration to the time required for sauna treatment. The substantial drop in training load (from day 20; Fig. 2d) upon commencing the sauna exposure period would be expected to cause a drop in PV over a few days that could be masked by the rapid initial increase in PV (e.g., days 19-24; Fig. 3a) that is known to occur with constant stress heat acclimation protocols. The effect of the sauna intervention on PV was unclear after the 
fourth exposure for reasons that could reflect the preceding reduction in training load now becoming evident against the background of the sauna intervention. Because the reductions in training load occurred after the initial clear increase in PV, we are confident changes in PV were primarily due to sauna exposure. Third, while the interrupted time series design we adopted may not be as strong as a crossover/parallel group design, we made comparisons with respect to the smallest worthwhile change, increasing the certainty of practical findings. Finally, the cyclists lived in a tropical environment and naturally exposed to high daily temperatures (Table 1) outside of the sauna, possibly exhibiting a greater thermal tolerance compared with others living in a cooler natural environment (Garrett et al. 2012; Garrett et al. 2009; Scoon et al. 2007). Therefore, it is possible that the physiological acclimation responses we observed may have been greater if the athletes were not moderately heat acclimatized. Nevertheless, the cyclists identified the sauna conditions as very uncomfortable and very hot, but tolerable.

\section{Conclusions}

The present study has demonstrated that sauna-bathing following normal training induced likely moderate-tolarge PV expansion in well-trained cyclists after just 4 exposures. The importance of these findings is that, postexercise sauna bathing may offer a time efficient means by which stimulate heat acclimation without substantially impacting on daily athlete training. However, consideration is required if such intervention is associated with a prolonged reduction in training load which may impact on actual performance. The value of HR-based measures to monitor changes in PV in this setting remains unclear. Further studies identifying the optimal protocol (i.e., repeating 3 days sauna, 1 day off) and associated training load management for maximising the potential benefits of sauna bathing for performance are also warranted.

\section{Acknowledgments}

The authors would like to thank the participants for their substantial commitment and assistance with the study. This study was funded by the Centre of Excellence for Applied Sport Science Research at the Queensland Academy of Sport.

\section{Ethical standards}

The current experiment was conducted in Australia with all activities complying with the current laws of Australia 


\section{Conflict of Interest}

Aaron Halliday was supported by a scholarship from the Centre of Excellence for Applied Sport Science Research at Queensland Academy of Sport. Dr Jamie Stanley is supported by research grant funding from the Centre of Excellence for Applied Sport Science Research at Queensland Academy of Sport. The remaining authors declare that they have no conflict of interest. 


\section{References}

Al Haddad H, Laursen PB, Chollet D, Ahmaidi S, Buchheit M (2011) Reliability of resting and postexercise heart rate measures. Int J Sports Med 32: 598-605

Armstrong LE, Maresh CM, Castellani JW, Bergeron MF, Kenefick RW, LaGasse KE, Riebe D (1994) Urinary indices of hydration status. Int J Sport Nutr 4: 265-279

Borresen J, Lambert M (2007) Changes in heart rate recovery in response to acute changes in training load. Eur J Appl Physiol 101: 503-511

Buchheit M (2014) Monitoring training status with HR measures: do all roads lead to Rome? Frontiers in Physiology 5

Buchheit M, Chivot A, Parouty J, Mercier D, Al Haddad H, Laursen P, Ahmaidi S (2010) Monitoring endurance running performance using cardiac parasympathetic function. Eur J Appl Physiol 108: 1153-1167

Buchheit M, Laursen P, Al Haddad H, Ahmaidi S (2009) Exercise-induced plasma volume expansion and postexercise parasympathetic reactivation. Eur J Appl Physiol 105: 471-481

Buchheit M, Papelier Y, Laursen PB, Ahmaidi S (2007) Noninvasive assessment of cardiac parasympathetic function: postexercise heart rate recovery or heart rate variability? Am J Physiol Heart Circ Physiol 293: H8-10

Buchheit M, Racinais S, Bilsborough JC, Bourdon PC, Voss SC, Hocking J, Cordy J, Mendez-Villanueva A, Coutts AJ (2013) Monitoring fitness, fatigue and running performance during a pre-season training camp in elite football players. J Sci Med Sport 16: 550-555

Buchheit M, Voss SC, Nybo L, Mohr M, Racinais S (2011) Physiological and performance adaptations to an inseason soccer camp in the heat: Associations with heart rate and heart rate variability. Scand J Med Sci Sports 21: e477-e485

Cohen J (1988) Statistical power analysis for the behavioral sciencies. Routledge, London, United Kingdom

Garrett A, Creasy R, Rehrer N, Patterson M, Cotter J (2012) Effectiveness of short-term heat acclimation for highly trained athletes. Eur J Appl Physiol 112: 1827-1837

Garrett A, Goosens N, Rehrer N, Patterson M, Cotter J (2009) Induction and decay of short-term heat acclimation. Eur J Appl Physiol 107: 659-670

Garrett A, Rehrer N, Patterson M (2011) Induction and decay of short-term heat acclimation in moderately and highly trained athletes. Sports Med 41: 757-771

Hopkins W (2000) Measures of Reliability in Sports Medicine and Science. Sports Med 30: 1-15

Hopkins W (2006) Spreadsheets for analysis of controlled trials, with adjustment for a subject characteristic. Sportscience 10: 46-50

Hopkins W, Marshall S, Batterham A, Hanin J (2009) Progressive statistics for studies in sports medicine and exercise science. Med Sci Sports Exerc 41: 3-13

Hopkins WG (2010) Linear models and effect magnitudes for research, clinical and practical applications. Sportscience 14: 49-57 (sportsci.org/2010/wghlinmod.htm)

Horowitz M, Peyser Y, Muhlrad A (1986a) Alterations in cardiac myosin isoenzymes distribution as an adaptation to chronic environmental heat stress in the rat. J Mol Cell Cardiol 18: 511-515

Horowitz M, Shimoni Y, Parnes S, Gotsman M, Hasin Y (1986b) Heat acclimation: cardiac performance of isolated rat heart. J Appl Physiol 60: 9-13 
Lamberts RP, Swart J, Noakes TD, Lambert MI (2011) A novel submaximal cycle test to monitor fatigue and predict cycling performance. Br J Sports Med 45: 797-804

Lorenzo S, Halliwill J, Sawka M, Minson C (2010) Heat acclimation improves exercise performance. J Appl Physiol 109: 1140-1147

Lundvall J, Lindgren P (1998) F-cell shift and protein loss strongly affect validity of PV reductions indicated by $\mathrm{Hb} / \mathrm{Hct}$ and plasma proteins. J Appl Physiol 84: 822-829

Morris S, Ruel M, Cohen R, Dewey K, de la Brière B, Hassan M (1999) Precision, accuracy, and reliability of hemoglobin assessment with use of capillary blood. Am J Clin Nutr 69: 1243-1248

Patterson M, Stocks J, Taylor N (2004) Sustained and generalized extracellular fluid expansion following heat acclimation. J Physiol 559: 327-334

Penttilä J, Helminen A, Jartti T, Kuusela T, Huikuri H, Tulppo M, Coffeng R, H. S (2001) Time domain, geometrical and frequency domain analysis of cardiac vagal outflow: effects of various respiratory patterns. Clin Physiol 21: 365-376

Plews DJ, Laursen PB, Stanley J, Kilding AE, Buchheit M (2013) Training Adaptation and Heart Rate Variability in Elite Endurance Athletes: Opening the Door to Effective Monitoring. Sports Med 43: 773-781

Racinais S, Buchheit M, Bilsborough J, Bourdon P, Cordy J, Coutts A (2014) Physiological and Performance Responses to a Training-Camp in the Heat in Professional Australian Football Players. Int J Sport Physiol Perform 9: 598-603

Ridge B, Pyke F (1986) Physiological responses to combinations of exercise and sauna. Aust J Sci Med Sport 18: $25-28$

Saitoh T, Ogawa Y, Aoki K, Shibata S, Otsubo A, Kato J, Iwasaki K-i (2008) Bell-shaped relationship between central blood volume and spontaneous baroreflex function. Auton Neurosci 143: 46-52

Sanchis-Gomar F, Cortell-Ballester J, Pareja-Galeano H, Banfi G, Lippi G (2013) Hemoglobin Point-of-Care Testing: The HemoCue System. Journal of Laboratory Automation 18: 198-205

Sandercock G, Bromley P, Brodie D (2005) Effects of exercise on heart rate variability: inferences from metaanalysis. Med Sci Sports Exerc 37: 433-439

Scoon GS, Hopkins W, Mayhew S, Cotter JD (2007) Effect of post-exercise sauna bathing on the endurance performance of competitive male runners. J Sci Med Sport 10: 259-262

Shirreffs S, Armstrong L, Cheuvront S (2004) Fluid and electrolyte needs for preparation and recovery from training and competition. J Sports Sci 22: 57-63

Stanley J, Peake J, Buchheit M (2013a) Consecutive days of cold water immersion: effects on cycling performance and heart rate variability. Eur J Appl Physiol 113: 371-384

Stanley J, Peake JM, Buchheit M (2013b) Cardiac Parasympathetic Reactivation Following Exercise: Implications for Training Prescription. Sports Med 43: 1259-1277

Strauss M, Davis R, Rosenbaum J, Rossmeisl E (1951) "Water diuresis" produced during recumbency by the intravenous infusion of isotonic saline solution. J Clin Invest 30: 862-868

Task Force of the European Society of Cardiology and the North American Society of Pacing and Electrophysiology (1996) Heart Rate Variability: Standards of Measurement, Physiological Interpretation, and Clinical Use. Eur Heart J 93: 1043-1065

Wallace L, Slattery K, Coutts A (2013) A comparison of methods for quantifying training load: relationships between modelled and actual training responses. Eur J Appl Physiol: 1-10 
Warburton D, Gledhill N, Quinney H (2000) Blood Volume, Aerobic Power, and Endurance Performance: Potential Ergogenic Effect of Volume Loading. Clin J Sport Med 10: 59-66

Wyndham CH, Benade AJ, Williams CG, Strydom NB, Goldin A, Heyns AJ (1968) Changes in central circulation and body fluid spaces during acclimatization to heat. J Appl Physiol 25: 586-593 